\title{
Isolation and Growth Characteristics of SARS-CoV-2 in Vero Cell
}

\author{
Pingping $\mathrm{Yao}^{1} \cdot \mathrm{Yachun}_{\mathrm{Zhang}}{ }^{2} \cdot$ Yisheng Sun $^{1} \cdot \mathrm{Yulin}_{\mathrm{Gu}}{ }^{2} \cdot$ Fang $\mathrm{Xu}^{1} \cdot \mathrm{Bo} \mathrm{Su}^{2} \cdot$ Chen $\mathrm{Chen}^{1} \cdot$ \\ Hangjing $\mathrm{Lu}^{1} \cdot$ Dehui Wang ${ }^{2} \cdot \mathrm{Zhangnv} \mathrm{Yang}^{1} \cdot$ Biao Niu $^{2} \cdot$ Jiancai Chen ${ }^{1} \cdot \mathrm{Lixia} \mathrm{Xie}^{2} \cdot$ Lei Chen $^{2}$. \\ Yajing Zhang ${ }^{2} \cdot$ Hui Wang ${ }^{2} \cdot$ Yuying Zhao ${ }^{2} \cdot$ Yue Guo $^{2} \cdot$ Juncheng Ruan ${ }^{2} \cdot$ Zhiyong Zhu $^{1} \cdot$ Zhenfang Fu $^{3,4}$ (D) \\ Dayong $\operatorname{Tian}^{2}$ (D) $\cdot \mathrm{Qi} \mathrm{An}^{2}$ (D) Jianmin Jiang ${ }^{1}$ (I) Hanping Zhu ${ }^{1}$ (D)
}

Received: 1 April 2020 / Accepted: 19 May 2020 / Published online: 19 June 2020

(c) Wuhan Institute of Virology, CAS 2020

\section{Dear Editors,}

The coronavirus disease 2019 (COVID-19), caused by SARS-CoV-2, broke out in early December 2019 has escalated into a global pandemic (Lai et al. 2020). Till the May 20th 2020, more than 4,700,000 people were infected and the number is still increasing especially in Europe, North America and Asia (https://covid19.who.int/). And there is an urgent need to understand the biology of SARS$\mathrm{CoV}-2$. Here we report the isolation and characterization of seven isolates of SARS-CoV-2 from seven patients. All these isolates were adapted to grow in Vero cells, and showed significant different replication characteristics. Sequence analysis revealed that these viruses shared

Pingping Yao and Yachun Zhang have contributed equally to this work

\footnotetext{
Hanping Zhu

hpzhu@cdc.zj.cn

$\triangle$ Jianmin Jiang

jmjiang@cdc.zj.cn

$\triangle$ Qi An

anqi@king-cell.com

$凶$ Dayong Tian

tiandayong@king-cell.com

$\triangle$ Zhenfang Fu

zhenfu@uga.edu

1 Key Lab of Vaccine, Prevention and Control of Infectious Disease of Zhejiang Province, Zhejiang Provincial Center for Disease Control and Prevention, Hangzhou 310051, China

2 R\&D Department, Shanghai King-Cell Biotechnology Co., Ltd., Shanghai 201506, China

3 State Key Laboratory of Agricultural Microbiology, Huazhong Agricultural University, Wuhan 430070, China

4 College of Veterinary Medicine, Huazhong Agricultural University, Wuhan 430070, China
}

similar sequences to reported SARS-CoV-2 strains, except for $8 \#$.

All of seven COVID-19 cases, from different cities of Zhejiang Province, were confirmed by real-time RT-PCR around 2020/01/26 (2020/01/24-2020/01/28). Basic information of those patients were listed in Table 1. Vero cells were seeded overnight in MEM containing 10\% FBS before samples (processed swabs or sputum) were inoculated. After incubation for an hour, the cells were replenished with fresh maintain medium (MEM containing 3\% FBS). The cells were observed daily for cytopathic effect (CPE) from the 2nd to the 7th days (Fig. 1A). At the day 7, the cell culture supernatant was collected for virus titration (Table 1).

It is well known that mutation on viral genes may have an impact on virulence, immunogenicity and other characterizations of viruses. In a recent report, 149 mutations were found among 103 sequenced isolates of SARS-CoV-2 (Tang et al. 2020). To investigate whether our viruses show mutations different from other reported SARS-CoV-2, all the seven isolates were sequenced at the 3rd passage and the sequences were aligned with coding sequence (CDS) of SARS-CoV-2 Wuhan-Hu-1 (NC_045512). Three and four of the seven isolates belong to the $\mathrm{L}$ and the $\mathrm{S}$ clades, respectively (Table 1) (Tang et al. 2020). It is interesting to note that these seven patients infected with SARS-CoV-2 have high viral load in the early stage of clinical sign, which is consistent with previous reports (Kim et al. 2020; Zou et al. 2020). As shown in Fig. 1B, genomic sequences of the viruses share sequence identity higher than $99.9 \%$. The phylogenetic analysis of the isolated viruses together with other available viruses indicates that the seven isolates are closely related to each other, as well as to the other published sequences, which is consistent with the previous studies (Lu et al. 2020; Ren et al. 2020; Zhou et al. 2020) (Fig. 1C). And, each virus has 2-5 mutations except for isolate 12\#, which is exactly the same as Wuhan-Hu-1. It's worth noting that apart from the 5 mutations, sequence of 
A
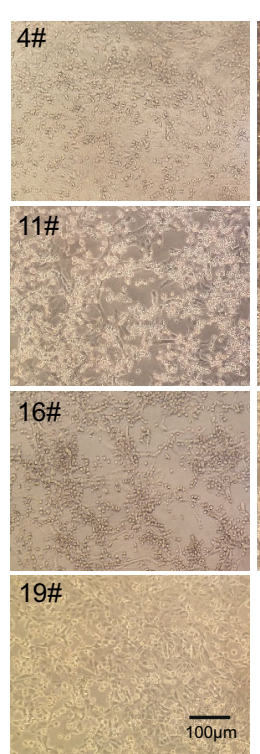

C

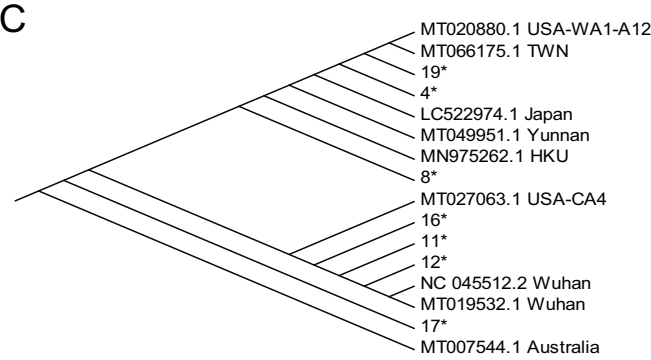

$E$

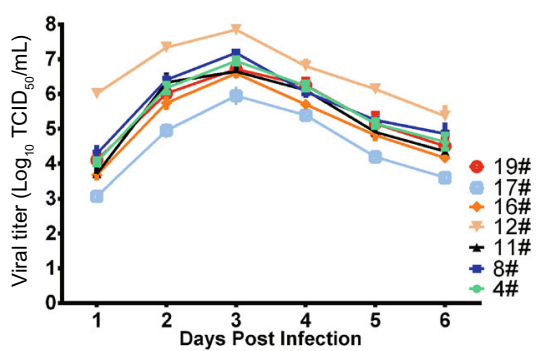

B

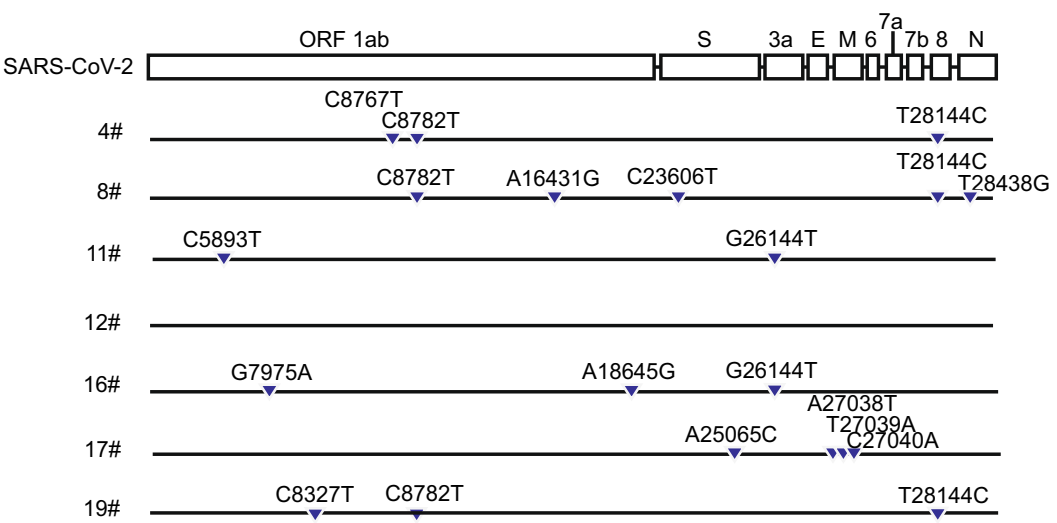

D

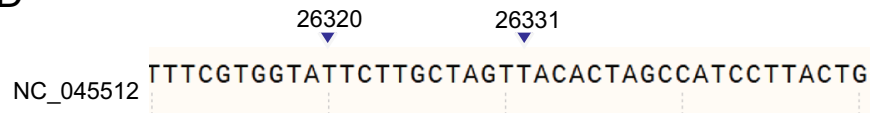

8\# P2

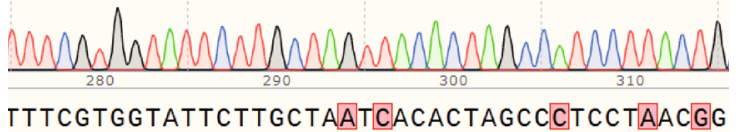

8\# P3

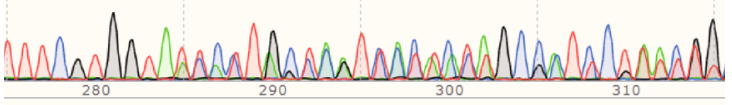

TTTCGTGGTA-....... ACACTAGCCATCCTTACTG

8\# P4

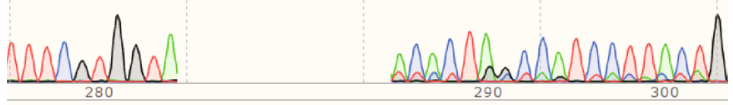

TTTCGTGGTA $\ldots \ldots$ ACACTAGCCATCCTTACTG

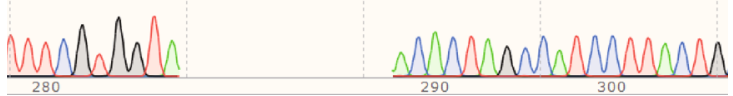

Fig. 1 Characteristics of SARS-CoV-2. A The cytopathic effect was observed in Vero cells infected with the isolated viruses at 5 dpi. B Nucleotide mutations of isolated viruses at passage 3. The SARSCoV-2 infected-cells were homogenized with Trizol, and total RNA was extracted and was used for RT-PCR as described below. Firststrand cDNA was synthesized with oligo(dT) primers, then the complete genome was segmented amplified and sequenced.

isolate 8\# shows obvious gene deletion at 26320-26331 (E. gene) (Fig. 1D, 8\# P3). To confirm this, isolate 8\# at passage 2 and 4 was sequenced (Fig. 1D, 8\# P2, 8\# P4). Sequencing result showed that the nesting peak gradually weakened from P2 to P4 (Fig. 1D). At P4, a 12-basedeficient virus was predominant. The in vitro and in vivo effect of the deletion on this isolate should be further studied. On the contrary, isolate $12 \#$ did not mutate temporarily (P3) during in vitro passage. These results indicate
Sequencing results were analyzed by SnapGene. C Phylogenetic tree was generated by Clustal Omega based on amino acid sequence of seven isolates and part of the available viruses. D Sequence results of 8\#, from Passage 2 to Passage 4. E The multiple growth curve of isolated viruses. The virus growth curves were drawn based on the viral titers measured at each indicated time point. The growth curves were obtained by three independent experiments.

that mutation occurred during virus passage in Vero cells, suggesting that virus purification and genetic monitoring are necessary in both vaccine development and etiology research.

To investigate the growth characteristics of seven isolated viruses, Vero cell was infected with each virus at a MOI of 0.001. The supernatant was collected for virus titration at 1, 2, 3, 4, 5, 6 days post infection (dpi). For the virus titration, Vero cells seeded in 96-well plate were 
Table 1 Basic information of the isolated strains.

\begin{tabular}{llllllll}
\hline Virus & Gender & Location & Confirmed time & Sample & Ct value & Clade & Titer $\left(\mathrm{TCID}_{50} / \mathrm{mL}\right)$ \\
\hline $4 \#$ & Female & Shaoxing & $2020 / 01 / 24$ & Pharyngeal swab & 22 & $\mathrm{~L}$ & 5.25 \\
$8 \#$ & Male & Hangzhou & $2020 / 01 / 26$ & Pharyngeal swab & 26 & $\mathrm{~L}$ & 5.0 \\
$11 \#$ & Male & Wenzhou & $2020 / 01 / 28$ & Sputum & 22 & $\mathrm{~S}$ & 5.25 \\
$12 \#$ & Male & Wenzhou & $2020 / 01 / 26$ & Sputum & 28 & $\mathrm{~S}$ & 6.25 \\
$16 \#$ & Male & Ningbo & $2020 / 01 / 24$ & Pharyngeal swab & 23 & $\mathrm{~S}$ & 5.0 \\
$17 \#$ & Female & Shaoxing & $2020 / 01 / 27$ & Pharyngeal swab & 26 & $\mathrm{~S}$ & 3.25 \\
$19 \#$ & Male & Huzhou & $2020 / 01 / 25$ & Pharyngeal swab & 24 & L & 6.25 \\
\hline
\end{tabular}

infected with serial tenfold diluted supernatant. CPE were observed and recorded at $96 \mathrm{~h}$ after infection. Virus titer was calculated by Reed-Muench method (Kint et al. 2015). As shown in Fig. 1E, all the seven isolated virus showed similar growth character: virus titers are detectable at $1 \mathrm{dpi}$, peak at $3 \mathrm{dpi}$, and then begin to decline. Among the seven isolates, the peak titer of isolate $12 \#$ reached $7.75 \mathrm{TCID}_{50} / \mathrm{mL}$, which is much higher than the other ones.

In conclusion, seven SARS-CoV-2 strains were isolated, sequenced and characterized in Vero cells, and a deletion mutation was identified after short passage in Vero cells. These results shall facilitate the understanding of the characteristics of SARS-CoV-2 in vitro.

Acknowledgements This work was partially supported by the National Natural Science Foundation of China (31702248).

\section{Compliance with Ethical Standards}

Conflict of interest The authors declare that they have no conflict of interest.

Animal and Human Rights Statement The study was approved by the Ethics Committees of Prevention and Control of Infectious Disease of Zhejiang Province. All participants provided written informed consent. Written consents were obtained from all parents involved in the study.

\section{References}

Kim JY, Ko JH, Kim Y, Kim YJ, Kim JM, Chung YS, Kim HM, Han MG, Kim SY, Chin BS (2020) Viral load kinetics of SARS$\mathrm{CoV}-2$ infection in first two patients in Korea. J Korean Med Sci 35:e86

Kint J, Maier HJ, Jagt E (2015) Quantification of infectious bronchitis coronavirus by titration in vitro and in ovo. Methods Mol Biol 1282:89-98

Lai CC, Shih TP, Ko WC, Tang HJ, Hsueh PR (2020) Severe acute respiratory syndrome coronavirus2 (SARS-CoV-2) and coronavirus disease-2019 (COVID-19): the epidemic and the challenges. Int J Antimicrob Agents 55:105924

Lu R, Zhao X, Li J, Niu P, Yang B, Wu H et al (2020) Genomic characterisation and epidemiology of 2019 novel coronavirus: implications for virus origins and receptor binding. Lancet 395:565-574

Ren LL, Wang YM, Wu ZQ, Xiang ZC, Guo L, Xu T et al (2020) Identification of a novel coronavirus causing severe pneumonia in human: a descriptive study. Chin. Med. J. (Engl.) 133:1015-1024

Tang X, Wu C, Li X, Song Y, Yao X, Wu X, Duan Y, Zhang H, Wang Y, Qian Z, Cui J, Lu J (2020) On the origin and continuing evolution of SARS-CoV-2. Natl. Sci. Rev. https://doi.org/10. 1093/nsr/nwaa036

Zhou P, Yang XL, Wang XG, Hu B, Zhang L, Zhang W et al (2020) A pneumonia outbreak associated with a new coronavirus of probable bat origin. Nature 579:270-273

Zou L, Ruan F, Huang M, Liang L, Huang H, Hong Z, Yu J, Kang M, Song Y, Xia J, Guo Q, Song T, He J, Yen HL, Peiris M, Wu J (2020) SARS-CoV-2 viral load in upper respiratory specimens of infected patients. N Engl J Med. https://doi.org/10.1056/NEJMc2001737 